Société d'histoire de la révolution de 1848 et des

révolutions du XIXe siècle

$63 \mid 2021$

Puissances de la Commune

Gilles DELLA-VEDOVA, La Montagne des possibles. Les acteurs du développement rural (Villard-de-Lans XIX ${ }^{e}$ XXI $I^{e}$ siècles)

Grenoble, Presses universitaires de Grenoble, 2020

Yann Decorzant

\title{
CpenEdition
}

Édition électronique

URL : https://journals.openedition.org/rh19/8072

DOI : $10.4000 /$ rh 19.8072

ISSN : $1777-5329$

Éditeur

La Société de 1848

Édition imprimée

Date de publication : 1 décembre 2021

Pagination : 242-245

ISSN : 1265-1354

Référence électronique

Yann Decorzant, «Gilles DELLA-VEDOVA, La Montagne des possibles. Les acteurs du développement rural (Villard-de-Lans xIxe-xxle siècles)", Revue d'histoire du XIXe siècle [En ligne], 63 | 2021, mis en ligne le 01 janvier 2022, consulté le 21 février 2022. URL : http://journals.openedition.org/rh19/8072 ; DOI : https://doi.org/10.4000/rh19.8072

Ce document a été généré automatiquement le 21 février 2022.

Tous droits réservés 


\section{Gilles DELLA-VEDOVA, La Montagne des possibles. Les acteurs du développement rural (Villard-de-Lans $\mathrm{XIX}^{e}-\mathrm{XXI}^{e}$ siècles)}

Grenoble, Presses universitaires de Grenoble, 2020

Yann Decorzant

\section{RÉFÉRENCE}

Gilles DELLA-VEDOVA, La Montagne des possibles. Les acteurs du développement rural (Villard-de-Lans XIXe-XXI e siècles), Grenoble, Presses universitaires de Grenoble, 2020, 528 p., 40,10 euros.

1 La lecture de l'ouvrage de Gilles Della-Vedova permet rapidement de prendre conscience de l'ampleur du travail accompli. Afin d'étudier la région de Villard-de-Lans sur plus d'un siècle, l'auteur a pris le parti de se saisir de son objet en croisant un nombre impressionnant de sources mais également de perspectives. En alliant une approche micro-historique avec une vision macro, en réalisant des biographies des acteurs et actrices chaque fois que cela a été possible et en intégrant des données quantitatives, il multiplie les prismes d'analyse afin de cerner au mieux son sujet. Grâce à ce minutieux travail, cet ouvrage, qui est tiré de sa thèse de doctorat, offre une vision très complète de l'histoire sociale et économique de cette région du Vercors durant la période choisie.

2 L'auteur a structuré son étude en deux parties chronologiques qui couvrent la période allant de 1830 à la fin des années 1920, avec une césure au milieu des années 1870. Dans la première partie, intitulée "Les faux-semblants d'un archaïsme rural» (p. 21), Gilles Della-Vedova se penche d'abord sur les différentes catégories d'activités socioéconomiques qui vont permettre de dresser un tableau de la région. Dans son premier 
chapitre, après une étude des sources laissées par le cultivateur Louis Mure-Ravaud, l'auteur effectue une description des produits phares émanant du canton (bois, lait, gants) avant de s'intéresser aux mouvements migratoires. Les diverses activités économiques précitées ont donné lieu à une économie pluriactive en mesure de s'intégrer dans les rouages du marché. Ainsi dans le cas de la ganterie, l'auteur relève que " [s] i celle-ci s'impose dans certains cas en raison d'une grande médiocrité des conditions d'existence, d'autres montrent que la couture des gants cherche à étayer le développement des individus et, à travers eux, des familles c'est-à-dire des exploitations dans lesquelles ils vivent » (p. 58-59). D'une manière générale, ce chapitre introductif cherche d'ailleurs à mettre en avant l'aspect dynamique du canton de Villard-de-Lans qui, loin de subir son environnement, en est en fait un des acteurs. Le deuxième chapitre est, lui, consacré spécifiquement à l'économie forestière, centrale dans la région, qui est étudiée tant au niveau de la gestion des propriétés que des usages des bois. Le dernier chapitre de cette première partie fait la part belle à l'étude de l'élevage des bovins dans le canton. L'auteur s'intéresse au développement de ce secteur durant la seconde partie du XIX ${ }^{e}$ siècle, aux tentatives qui sont faites pour l'améliorer, entre autres par la mise en place de la station d'élevage de Villard-de-Lans, ainsi qu'aux acteurs qui ont cherché à faire émerger une race bovine locale.

3 La seconde partie explore le développement de la région de la crise économique de la fin $\mathrm{du} \mathrm{XIX}^{\mathrm{e}}$ siècle à la décennie qui suit la Première Guerre mondiale. Ce choix chronologique se justifie d'une part car, outre les événements conjoncturels majeurs comme la crise de l'agriculture des années 1870 et 1880, plusieurs événements régionaux, notamment la création de la station d'élevage en 1875 ou le classement de Villard-de-Lans comme station climatique en 1930, constituent des tournants dans la trajectoire économique régionale. L'auteur explique d'ailleurs que son travail participe aux « relectures des temporalités qui cherchent à s'éloigner des ruptures politiques, en particulier de part et d'autre de la Grande Guerre, pour embrasser d'un œil neuf la scansion qui va des années 1880 aux années $1920 »$ (p. 159).

4 Le premier chapitre de cette partie s'inscrit dans la continuité du précédent car l'auteur poursuit son étude sur le thème de l'élevage et de la zootechnie. Ce chapitre intitulé "Une race inachevée pour un concours entravé ", est abordé en utilisant comme porte d'entrée la mise en place et la trajectoire du concours spécial de la race de Villard-de-Lans qui s'est tenu de 1893 à 1914. Dans le chapitre suivant, l'auteur se penche, tour à tour, sur l'évolution du canton durant la guerre, sur l'évolution de sa population et sur celle de son agriculture. Ensuite, il remet la focale sur les questions bovine et laitière qui servent de prisme à l'étude des changements structurels régionaux. Enfin, le rôle des femmes dans le développement économique local est spécifiquement interrogé, que ce soit dans le cadre de l'activité gantière, la mise en nourrice ou, plus largement, dans différentes sphères productives où les femmes sont très présentes. Le dernier chapitre du livre, "L'émergence d'un système touristique " permet à l'auteur de terminer son cheminement le long des transformations socioéconomiques du canton de Villard-de-Lans. En étudiant l'arrivée des premiers touristes et le développement des voies de communications, puis la mise en place des structures d'hébergement et, enfin, le positionnement de la région par rapport au traitement des malades atteints de tuberculose, Gilles Della-Vedova pose les bases qui permettent de comprendre l'évolution future du canton. 
5 Plusieurs grandes problématiques se mêlent dans le livre. Une première interroge la prétendue stagnation économique des espaces ruraux de montagnes qui s'opposeraient à l'arrivée d'une forme de modernité et de développement. Découlant de cela, se pose la question de l'isolement des territoires de montagne et de leur imperméabilité aux circulations régionales, nationales voire internationales. Dans le cas qui nous intéresse, l'auteur cherche donc à déterminer si le canton de Villard-de-Lans et ses environs, bien que situés en zone de montagne, sont des territoires reclus, coupés des circulations et des mouvements extérieurs. Pour répondre à cette interrogation, Gilles Della-Vedova aborde les multiples thématiques de sa recherche en gardant ce fil rouge sur l'ensemble de la période qu'il étudie. Il en arrive à la conclusion que, loin d'être isolés, les territoires du canton de Villard-de-Lans sont intégrés dans des réseaux de circulation des personnes, des produits, des idées et des évolutions économiques de l'époque. Cependant, ils les intègrent à leur propre rythme, sans les subir de façon incontrôlée, cette observation inscrivant l'étude de Della Vedova dans un courant historiographique où on retrouve les travaux d'Anne-Marie Granet-Abisset, de Pierre Judet ou encore, pour n'en citer que quelques-uns, de Luigi Lorenzetti.

6 Dans ce cadre général, Gilles Della-Vedova questionne nombre de thématiques sousjacentes. À titre d'exemples, quand il se penche sur la question du bois, il inclut dans son étude le thème plus large de la gestion des communs, très sensible par ailleurs dans le canton. Dans les parties qu'il consacre à l'élevage, l'auteur étudie également en détail le rôle des institutions, des notables et, inversement, celui des petits propriétaires, celui de l'État, les implications politiques ou encore les réseaux d'échanges avec d'autres régions de France ou de Suisse. Lorsqu'il aborde la naissance du tourisme et la mise en place progressive des premières structures d'hébergement, il questionne les dynamiques endogènes et exogènes de ce mouvement en regardant aussi bien les données quantitatives que les trajectoires individuelles des personnes qui sont actives dans le processus. Avec ces quelques exemples, c'est la richesse de ce travail que l'on cherche à mettre en évidence. En effet, à travers les multiples questions qu'il aborde, Gilles Della-Vedova suggère des liens vers d'autres problématiques ou ouvre des portes sur d'autres questionnements sous-jacents, ce qui rend la lecture de cet ouvrage des plus intéressants.

$7 \quad$ La Montagne des possibles est une étude très complète et précise de l'histoire de la région de Villard-de-Lans qui intègre son objet dans une perspective nettement plus large. Les allers-retours permanents que fait l'auteur entre les différentes échelles d'analyse permettent de bien saisir les multiples enjeux qui marquent la trajectoire de la modernisation de la région, tout en offrant une histoire régionale très détaillée et construite sur une quantité et une diversité de sources notable. Peut-être peut-on juste se demander si des comparaisons plus fréquentes avec des études consacrées à des régions similaires en France ou ailleurs dans l'arc alpin n'auraient pas renforcé encore ce jeu d'échelle. Outre l'apport historiographique de ce travail, les méthodes utilisées par Gilles Della-Vedova semblent particulièrement inspirantes pour l'étude des zones rurales de montagne. Ainsi, la mise en perspective constante des grandes tendances du développement économique de la région avec les données tirées des biographies individuelles et familiales semble particulièrement féconde. Elle permet d'incarner ce mouvement, de comprendre, sur la durée, les rôles des actrices et des acteurs, et, surtout, d'en montrer toute la complexité. À ce titre, l'étude du concours spécial de la race bovine de Villard-de-Lans met particulièrement bien en lumière l'apport de cette 
méthode. Pour terminer, on ne peut donc que recommander la lecture de ce livre pour son apport à la connaissance et à la compréhension de la région étudiée, mais également pour les perspectives méthodologiques et problématiques qu'il propose. 